\title{
An Algebraic Approach to Practical and Scalable Overlay Network Monitoring
}

\author{
Yan Chen \\ Department of Computer Science \\ Northwestern University \\ ychen@cs.northwestern.edu
}

\author{
David Bindel, Hanhee Song, Randy Katz \\ Division of Computer Science \\ University of California at Berkeley \\ (dbindel@cs, coolcoon@uclink, \\ randy@cs).berkeley.edu
}

\begin{abstract}
Overlay network monitoring enables distributed Internet applications to detect and recover from path outages and periods of degraded performance within seconds. For an overlay network with $n$ end hosts, existing systems either require $O\left(n^{2}\right)$ measurements, and thus lack scalability, or can only estimate the latency but not congestion or failures. Our earlier extended abstract [1] briefly proposes an algebraic approach that selectively monitors $k$ linearly independent paths that can fully describe all the $O\left(n^{2}\right)$ paths. The loss rates and latency of these $k$ paths can be used to estimate the loss rates and latency of all other paths. Our scheme only assumes knowledge of the underlying IP topology, with links dynamically varying between lossy and normal.

In this paper, we improve, implement and extensively evaluate such a monitoring system. We further make the following contributions: i) scalability analysis indicating that for reasonably large $n$ (e.g., 100), the growth of $k$ is bounded as $O(n \log n)$, ii) efficient adaptation algorithms for topology changes, such as the addition or removal of end hosts and interconnections, iii) measurement load balancing schemes, iv) topology measurement error handling, and v) design and implementation of an adaptive streaming media system as a representative application. Both simulation and Internet experiments demonstrate we obtain highly accurate path loss rate estimation while adapting to topology changes within seconds and handling topology errors.
\end{abstract}

\section{Categories and Subject Descriptors}

C.2.3 [Network Operations]: Network monitoring

\section{General Terms}

Algorithms, Measurement

\section{Keywords}

Overlay, Network measurement and monitoring, Numerical linear algebra, Scalability, Dynamics, Load balancing

Permission to make digital or hard copies of all or part of this work for personal or classroom use is granted without fee provided that copies are not made or distributed for profit or commercial advantage and that copies bear this notice and the full citation on the first page. To copy otherwise, to republish, to post on servers or to redistribute to lists, requires prior specific permission and/or a fee.

SIGCOMM'04, Aug. 30-Sept. 3, 2004, Portland, Oregon, USA.

Copyright 2004 ACM 1-58113-862-8/04/0008 ...\$5.00

\section{INTRODUCTION}

The rigidity of the Internet architecture makes it extremely difficult to deploy innovative disruptive technologies in the core. This has led to extensive research into overlay and peer-to-peer systems, such as overlay routing and location, application-level multicast, and peer-to-peer file sharing. These systems flexibly choose their communication paths and targets, and thus can benefit from end-to-end network distance estimation (e.g., latency and loss rate).

Accurate loss rate monitoring can detect path outages and periods of degraded performance within seconds. It can both facilitate distributed system management (such as a virtual private network (VPN) or a content distribution network), and help build adaptive overlay applications, e.g., streaming media as we show in [2].

Thus it is desirable to have a scalable overlay loss rate monitoring system which is accurate and incrementally deployable. However, existing network distance estimation systems are insufficient for this end. They can be grouped into two categories based on the targeted metrics: general metrics [3] and latency only [4, 5, 6, 7]. Systems in the former category can measure any metric, but require $O\left(n^{2}\right)$ measurements where $n$ is the number of end hosts, and thus lack scalability. On the other hand, the latency estimation systems are scalable, but cannot provide accurate congestion/failure detection (see Sec. 2).

We formulate the problem as follows: consider an overlay network of $n$ end hosts; we define a path to be a routing path between a pair of end hosts, and a link to be an IP link between routers. A path is a concatenation of links. There are $O\left(n^{2}\right)$ paths among the $n$ end hosts, and we wish to select a minimal subset of paths to monitor so that the loss rates and latencies of all other paths can be inferred.

In an earlier extended abstract [1], we sketched the idea of a tomography-based overlay monitoring system (TOM) in which we selectively monitor a basis set of $k$ paths. Any end-to-end path can be written as a unique linear combination of paths in the basis set. Consequently, by monitoring loss rates for the paths in the basis set, we infer loss rates for all end-to-end paths. This can also be extended to other additive metrics, such as latency. The end-to-end path loss rates can be computed even when the paths contain unidentifiable links for which loss rates cannot be computed.

In [1], we only briefly introduce the basic formulation and model. The following questions remain:

- How scalable is the system? In other words, how will $k$ grow as a function of $n$ ? It is our conjecture that for reasonably large $n$ (say 100), $k=O(n \log n)$ [1].

- In an overlay network, end hosts frequently join/leave 
the overlay and routing changes occur from time to time. How can the system adapt to these efficiently?

- How should the system distribute the measurement load among end hosts to improve scalability?

- How can the system maintain accuracy when there are topology measurement errors?

- How does TOM perform under various topologies and loss conditions as well as the real Internet?

To address these issues, in this paper, we make the following contributions.

- We show that $k$ does grow as $O(n \log n)$ through linear regression tests on various synthetic and real topologies. We also provide some explanation based on the Internet topology and the AS hierarchy.

- We design incremental algorithms for path addition and deletion which only cost $O\left(k^{2}\right)$ time instead of $O\left(n^{2} k^{2}\right)$ for complete reinitializion.

- We propose randomized schemes for measurement load balancing.

- We design effective schemes to handle topology measurement errors.

- We evaluate TOM through extensive simulations, as well as Internet experiments to validate the simulation.

Both simulation and PlanetLab experiments results show that we achieve high accuracy when estimating path loss rates with $O(n \log n)$ measurements. For the PlanetLab experiments, the average absolute error of loss rate estimation is only 0.0027 , and the average error factor is 1.1 , even though about $10 \%$ of the paths have no or incomplete routing information. The average setup (monitoring path selection) time is 0.75 second, and the online update of the loss rates for all 2550 paths takes only 0.16 second. In addition, we adapt to topology changes within seconds without sacrificing accuracy. The measurement load balancing reduces the load variation and the maximum vs. mean load ratio significantly, by up to a factor of 7.3 .

The rest of the paper is organized as follows. We survey related work in Sec. 2, describe our model and basic static algorithms in Sec. 3, and evaluate scalability in Sec. 4. We extend the algorithms to adapt to topology changes in Sec. 5, and to handle overload and topology measurement errors in Sec. 6. The methodology and results of simulation are described in Sec. 7, and those of Internet experiments are presented in Sec. 8. Finally, we conclude in Sec. 9.

\section{RELATED WORK}

There are many existing scalable end-to-end latency estimation schemes, which can be broadly classified into clusteringbased $[6,7]$ and coordinate-based systems $[4,5]$. Clusteringbased systems cluster end hosts based on their network proximity or latency similarity under normal conditions, then choose the centroid of each cluster as the monitor. But a monitor and other members of the same cluster often take different routes to remote hosts. So the monitor cannot detect congestion for its members. Similarly, the coordinates assigned to each end host in the coordinate-based approaches cannot embed any congestion/failure information.

Network tomography has been well studied ([8] provides a good survey). Most tomography systems assume limited measurements are available (often in a multicast tree-like structure), and try to infer link characteristics $[9,10]$ or shared congestion [11] in the middle of the network. However, the problem is under-constrained: there exist unidentifiable links [9] with properties that cannot be uniquely de-

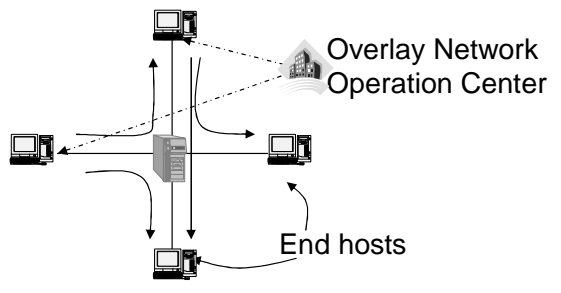

Figure 1: Architecture of a TOM system.

termined. In contrast, we are not concerned about the characteristics of individual links, and we do not restrict the paths we measure.

Shavitt, et al. also use algebraic tools to compute distances that are not explicitly measured [12]. Given certain "Tracer" stations deployed and some direct measurements among the Tracers, they search for path or path segments whose loss rates can be inferred from these measurements. Thus their focus is not on Tracer/path selection.

Recently, Ozmutlu, et al. selected a minimal subset of paths to cover all links for monitoring, assuming link-by-link latency is available via end-to-end measurement [13]. But the link-by-link latency obtained from traceroute is often inaccurate. And their approach is not applicable for loss rate because it is difficult to estimate link-by-link loss rates from end-to-end measurement. A similar approach was taken for selecting paths to measure overlay network [14]. The minimal set cover selected can only gives bounds for metrics like latency, and there is no guarantee as to how far the bounds are from the real values.

Furthermore, none of the existing work examines topology change, topology measurement errors, or measurement load balancing problems.

\section{MODEL AND BASIC ALGORITHMS}

\subsection{Algebraic Model}

Suppose there are $n$ end hosts that belong to a single or confederated overlay network(s). They cooperate to share an overlay monitoring service, and are instrumented by a central authority (e.g., an overlay network operation center (ONOC)) to measure the routing topology and path loss rates as needed ${ }^{1}$. For simplicity, we usually assume symmetric routing and undirected links in this paper. However, our techniques work without change for asymmetric routing, as evidenced in the PlanetLab experiments. Fig. 1 shows a sample overlay network with four links and four end hosts; six possible paths connect the end hosts. The end hosts measure the topology and report to the ONOC, which selects four paths and instruments two of the end hosts to measure the loss rates of those paths. The end hosts periodically report the measured loss rates to the ONOC. Then the ONOC infers the loss rates of every link, and consequently the loss rates of the other two paths. Applications can query the ONOC for the loss rate of any path, or they can set up triggers to receive alerts when the loss rates of paths of interest exceed a certain threshold [2].

We now introduce an algebraic model which applies to any network topology. Suppose an overlay network spans $s$ IP links. We represent a path by a column vector $v \in$ $\{0,1\}^{s}$, where the $j$ th entry $v_{j}$ is one if link $j$ is part of the

\footnotetext{
${ }^{1}$ As part of the future work, we will investigate techniques to distribute the work of the central authority.
} 


\begin{tabular}{|l|l|}
\hline Symbols & Meanings \\
\hline$M$ & total number of nodes \\
\hline$N$ & number of end hosts \\
\hline$n$ & number of end hosts on the overlay \\
\hline$r=O\left(n^{2}\right)$ & number of end-to-end paths \\
\hline$s$ & \# of IP links that the overlay spans on \\
\hline$t$ & number of identifiable links \\
\hline$G \in\{0,1\}^{r \times s}$ & original path matrix \\
\hline$G \in\{0,1\}^{k \times s}$ & reduced path matrix \\
\hline$k \leq s$ & rank of $G$ \\
\hline$l_{i}$ & loss rate on $i$ th link \\
\hline$p_{i}$ & loss rate on $i$ th measurement path \\
\hline$x_{i}$ & log(1-l $\left.l_{i}\right)$ \\
\hline$b_{i}$ & log(1- $\left.p_{i}\right)$ \\
\hline$v$ & vector in $\{0,1\}^{s}$ (represents path) \\
\hline$p$ & loss rate along a path \\
\hline $\mathcal{N}(G)$ & null space of $G$ \\
\hline $\mathcal{R}\left(G^{T}\right)$ & row(path) space of $G\left(==\operatorname{range}\left(G^{T}\right)\right)$ \\
\hline
\end{tabular}

\section{Table 1: Table of notations}

path, and zero otherwise. Suppose link $j$ drops packets with probability $l_{j}$; then the loss rate $p$ of a path represented by $v$ is given by

$$
1-p=\prod_{j=1}^{s}\left(1-l_{j}\right)^{v_{j}}
$$

Equation (1) assumes that packet loss is independent among links. Caceres et al. argue that the diversity of traffic and links makes large and long-lasting spatial link loss dependence unlikely in a real network such as the Internet [15]. Furthermore, the introduction of Random Early Detection (RED) [16] policies in routers will help break such dependence. In addition to [15], formula (1) has also rendered useful in many other link/path loss inference works [10, 9, 17, 14]. Our Internet experiments also show that the link loss dependence has little effect on the accuracy of (1).

We take logarithms on both sides of (1). Then by defining a column vector $x \in \mathbb{R}^{s}$ with elements $x_{j}=\log \left(1-l_{j}\right)$, and writing $v^{T}$ for the transpose of the column vector $v$, we can rewrite (1) as follows:

$$
\log (1-p)=\sum_{j=1}^{s} v_{j} \log \left(1-l_{j}\right)=\sum_{j=1}^{s} v_{j} x_{j}=v^{T} x
$$

There are $r=O\left(n^{2}\right)$ paths in the overlay network, thus $r$ linear equations of the form (2). Putting them together, we form a rectangular matrix $G \in\{0,1\}^{r \times s}$. Each row of $G$ represents a path in the network: $G_{i j}=1$ when path $i$ contains link $j$, and $G_{i j}=0$ otherwise. Let $p_{i}$ be the endto-end loss rate of the $i$ th path, and let $b \in \mathbb{R}^{r}$ be a column vector with elements $b_{i}=\log \left(1-p_{i}\right)$. Then we write the $r$ equations in form (2) as

$$
G x=b
$$

Normally, the number of paths $r$ is much larger than the number of links $s$. Equation (3) may be illustrated as in Fig. 2(a). This suggests that if we can select $s$ paths to monitor, and then solve the link loss rate variables $x$, the loss rate of other paths can be inferred from (3).

However, in general, $G$ is rank deficient: i.e., $k=\operatorname{rank}(G)$ and $k<s$. If $G$ is rank deficient, we will be unable to determine the loss rate of some links from (3). These links are also called unidentifiable in network tomography literature [9].

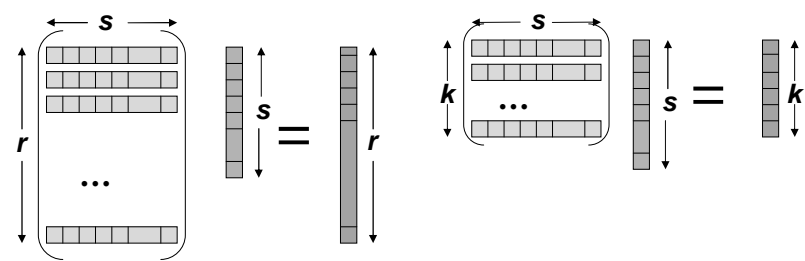

(a) $G x=b$

(b) $\bar{G} x_{G}=\bar{b}$

Figure 2: Matrix size representations.

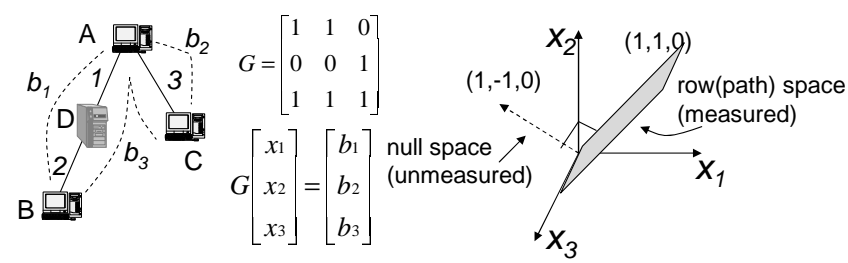

Figure 3: Sample overlay network.

Fig. 3 illustrates how rank deficiency can occur. There are three end hosts (A, B and C) on the overlay, three links (1, 2 and 3) and three paths between the end hosts. We cannot uniquely solve $x_{1}$ and $x_{2}$ because links 1 and 2 always appear together. We know their sum, but not their difference.

Viewing the geometry of the linear system in Fig. 3 with each variable $x_{i}$ as a dimension, there is a set of vectors $\alpha\left[\begin{array}{lll}1 & -1 & 0\end{array}\right]^{T}$ which are not defined by (3). This set of vectors is the null space of $G(\mathcal{N}(G))$. Meanwhile, there is an orthogonal row(path) space of $G\left(\mathcal{R}\left(G^{T}\right)\right)$, which for this example is a plane determined by $x_{1}+x_{2}$ and $x_{3}$. Unlike the null space, any vector on the row space can be uniquely determined by (3).

To separate the identifiable and unidentifiable components of $x$, we decompose $x$ as $x=x_{G}+x_{N}$, where $x_{G} \in \mathcal{R}\left(G^{T}\right)$ is its projection on the row space and and $x_{N} \in \mathcal{N}(G)$ is its projection on the null space (i.e., $G x_{N}=0$ ). The decomposition of $\left[\begin{array}{lll}x_{1} & x_{2} & x_{3}\end{array}\right]^{T}$ for the sample overlay is shown below.

$$
\begin{gathered}
x_{G}=\frac{\left(x_{1}+x_{2}\right)}{2}\left[\begin{array}{l}
1 \\
1 \\
0
\end{array}\right]+x_{3}\left[\begin{array}{l}
0 \\
0 \\
1
\end{array}\right]=\left[\begin{array}{c}
b_{1} / 2 \\
b_{1} / 2 \\
b_{2}
\end{array}\right] \\
x_{N}=\frac{\left(x_{1}-x_{2}\right)}{2}\left[\begin{array}{c}
1 \\
-1 \\
0
\end{array}\right]
\end{gathered}
$$

Thus the vector $x_{G}$ can be uniquely identified, and contains all the information we can know from (3) and the path measurements. The intuition of our scheme is illustrated through virtual links in [1].

Because $x_{G}$ lies in the $k$-dimensional space $\mathcal{R}\left(G^{T}\right)$, only $k$ independent equations of the $r$ equations in (3) are needed to uniquely identify $x_{G}$. We measure these $k$ paths to compute $x_{G}$. Since $b=G x=G x_{G}+G x_{N}=G x_{G}$, we can compute all elements of $b$ from $x_{G}$, and thus obtain the loss rate of all other paths. Next, we present more detailed algorithms.

\subsection{Basic Static Algorithms}

The basic algorithms involve two steps. First, we select a basis set of $k$ paths to monitor. Such selection only needs to be done once at setup. Then, based on continuous monitoring of the selected paths, we calculate and update the loss rates of all other paths. 


\subsubsection{Measurement Paths Selection}

To select $k$ linearly independent paths from $G$, we use standard rank-revealing decomposition techniques [18], and obtain a reduced system:

$$
\bar{G} x_{G}=\bar{b}
$$

where $\bar{G} \in \mathbb{R}^{k \times s}$ and $\bar{b} \in \mathbb{R}^{k}$ consist of $k$ rows of $G$ and $b$, respectively. The equation is illustrated in Fig. 2(b) (compared with $G x=b$ ).

As shown below, our algorithm is a variant of the QR decomposition with column pivoting [18, p.223]. It incrementally builds a decomposition $\bar{G}^{T}=Q R$, where $Q \in \mathbb{R}^{s \times k}$ is a matrix with orthonormal columns and $R \in \mathbb{R}^{k \times k}$ is upper triangular.

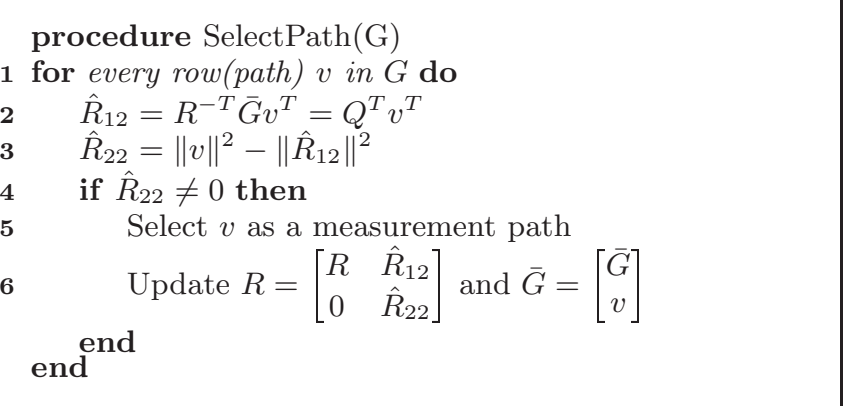

Algorithm 1: Path (row) selection algorithm

In general, the $G$ matrix is very sparse; that is, there are only a few nonzeros per row. We leverage this property for speedup. We further use optimized routines from the LAPACK library [19] to implement Algorithm 1 so that it inspects several rows at a time. The complexity of Algorithm 1 is $O\left(r k^{2}\right)$, and the constant in the bound is modest. The memory cost is roughly $k^{2} / 2$ single-precision floating point numbers for storing the $R$ factor. Notice that the path selection only needs to be executed once for initial setup.

\subsubsection{Path Loss Rate Calculations}

To compute the path loss rates, we must find a solution to the underdetermined linear system $\bar{G} x_{G}=\bar{b}$. The vector $\bar{b}$ comes from measurements of the paths. Zhang et al. report that path loss rates remain operationally stable in the time scale of an hour [20], so these measurements need not be taken simultaneously.

Given measured values for $\bar{b}$, we compute a solution $x_{G}$ using the QR decomposition we constructed during measurement path selection $[18,21]$. We choose the unique solution $x_{G}$ with minimum possible norm by imposing the constraint $x_{G}=\bar{G}^{T} y$ where $y=R^{-1} R^{-T} \bar{b}$. Once we have $x_{G}$, we can compute $b=G x_{G}$, and from there infer the loss rates of the unmeasured paths. The complexity for this step is only $O\left(k^{2}\right)$. Thus we can update loss rate estimates online, as verified in Sec. 7.4 and 8.2.

\section{SCALABILITY ANALYSIS}

An overlay monitoring system is scalable only when the size of the basis set, $k$, grows relatively slowly as a function of $n$. Given that the Internet has moderate hierarchical structure [22, 23], we proved that the number of end hosts is no less than half of the total number of nodes in the Internet. Furthermore, we proved that when all the end hosts are on the overlay network, $k=O(n)[1]$.
But what about if only a small fraction of the end hosts are on the overlay? Because $G$ is an $r$ by $s$ matrix, $k$ is bounded by the number of links $s$. If the Internet topology is a strict hierarchy like a tree, $s=O(n)$, thus $k=O(n)$. But if there is no hierarchy at all (e.g. a clique), $k=O\left(n^{2}\right)$ because all the $O\left(n^{2}\right)$ paths are linearly independent. Tangmunarunkit et al. found that the power-law degree Internet topology has moderate hierarchy [22]. It is our conjecture that $k=O(n \log n)$.

In this section, we will show through linear regression on both synthetic and real topologies that $k$ is indeed bounded by $O(n \log n)$ for reasonably large $n(e . g, 100)$. We explain it based on the power-law degree distribution of the Internet topology and the AS (Autonomous System) hierarchy.

We experiment with three types of BRITE [24] routerlevel topologies - Barabasi-Albert, Waxman and hierarchical models - as well as with a real router topology with 284,805 nodes [25]. For hierarchical topologies, BRITE first generates an autonomous system (AS) level topology with a Barabasi-Albert model or a Waxman model. Then for each AS, BRITE generates the router-level topologies with another Barabasi-Albert model or Waxman model. So there are four types of possible topologies. We show one of them as an example because they all have similar trends (see [2] for complete results).

We randomly select end hosts which have the least degree (i.e., leaf nodes) to form an overlay network. We test by linear regression of $k$ on $O(n), O(n \log n), O\left(n^{1.25}\right), O\left(n^{1.5}\right)$, and $O\left(n^{1.75}\right)$. As shown in Fig. 4, results for each type of topology are averaged over three runs with different topologies for synthetic ones and with different random sets of end hosts for the real one. We find that for Barabasi-Albert, Waxman and real topologies, $O(n)$ regression has the least residual errors - actually $k$ even grows slower than $O(n)$. The hierarchical models have higher $k$, and most of them have $O(n \log n)$ as the best fit. Conservatively speaking, we have $k=O(n \log n)$.

Note that such trend still holds when the end hosts are sparsely distributed in the Internet, e.g., when each end host is in a different access network. One extreme case is the "star" topology - each end host is connected to the same center router via its own access network. In such a topology, there are only $n$ links. Thus $k=O(n)$. Only topologies with very dense connectivity, like a full clique, have $k=O\left(n^{2}\right)$. Those topologies have little link sharing among the end-toend paths.

The key observation is that when $n$ is sufficiently large, such dense connectivity is very unlikely to exist in the Internet because of the power-law degree distribution. Tangmunarunkit et al. found that link usage, as measured by the set of node pairs (source-destination pairs) whose traffic traverses the link, also follows a power-law distribution, i.e., there is a very small number of links that are on the shortest paths of the majority of node pairs. So there is significant amount of link sharing among the paths, especially for backbone links, customer links, and peering links.

Such link sharing can easily lead to rank deficiency of the path matrix for overlay networks. As an example, consider an overlay within a single AS. The AS with the largest number of links (exclusive of customer and peering links) in [26] has 5,300 links. Even considering the coverage factor $(55.6 \%$ as in Table 2 of [26]), there are at most 9,600 links. Since there are $n(n-1)$ paths among $n$ nodes, link sharing must occur before $n=100$; in fact, substantial link sharing is 


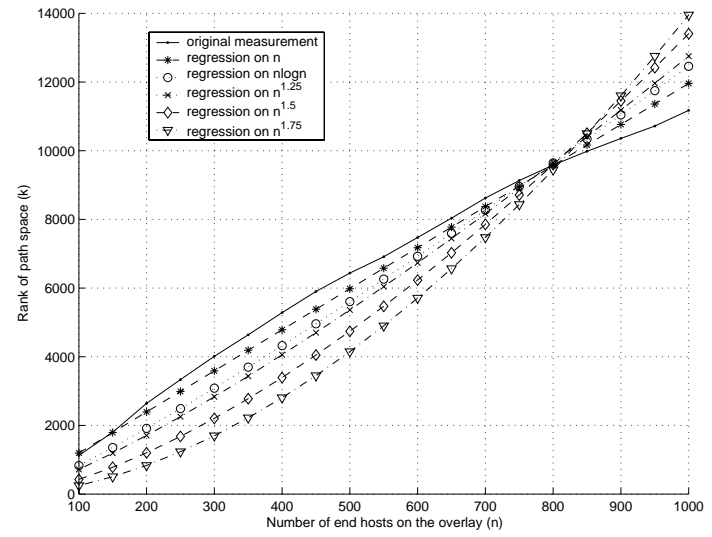

Barabasi-Albert model of 20K nodes

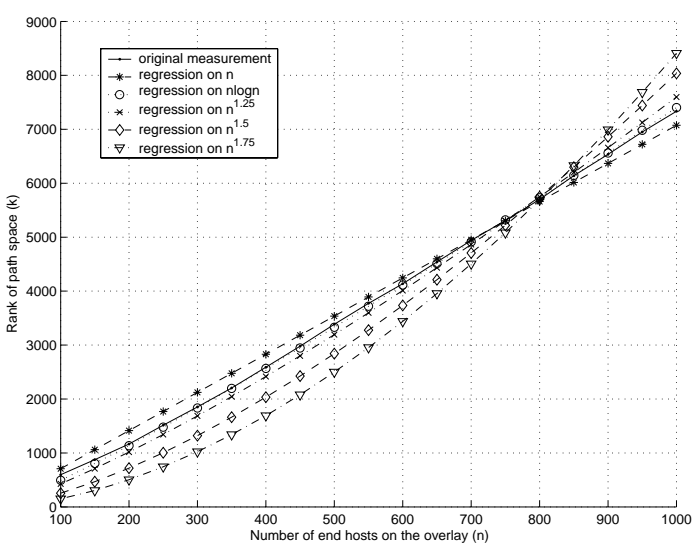

Hierarchical model of $20 \mathrm{~K}$ nodes

(AS-level: Barabasi-Albert and router level: Waxman)

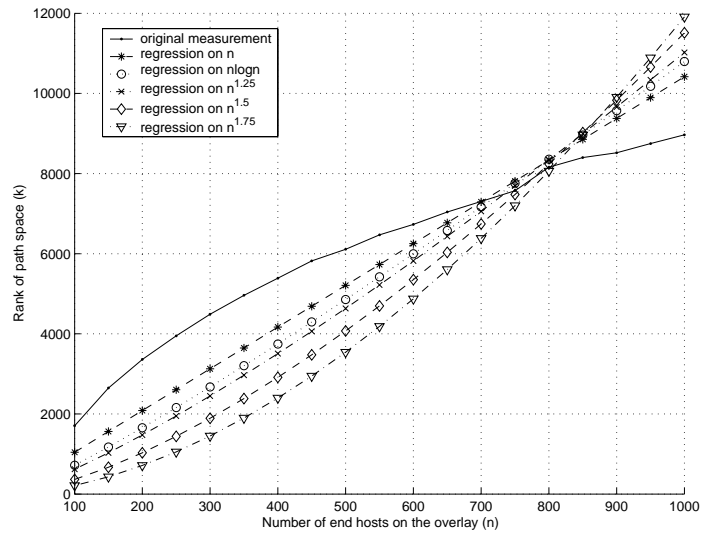

Waxman model of $10 \mathrm{~K}$ nodes

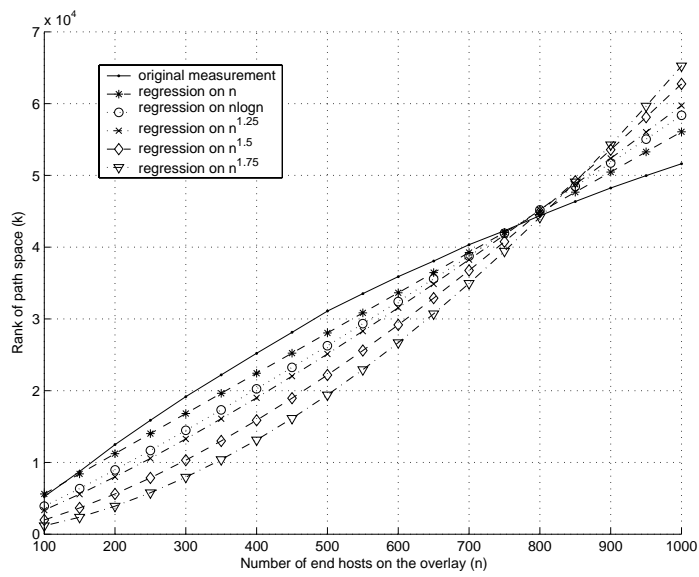

A real topology of 284,805 routers

Figure 4: Regression of $k$ in various functions of $n$ under different router-level topologies.

likely to occur for even smaller $n$.

Now consider an overlay network that spans two ASes connected by $y$ customer/peering links, with $n / 2$ nodes in one AS and $n / 2$ nodes in the other. The $n^{2} / 2$ cross-AS paths can be modelled as linear combination of $2 y \times n+2 y$ virtual links - bi-directional links from each end host to its $y$ peering link routers, and $y$ bi-directional peering links. Thus given $y$ is normally much less than $n$ and can be viewed as a constant, only $O(n)$ paths need to be measured for the $O\left(n^{2}\right)$ cross-AS paths.

Now consider an overlay on multiple ASes. According to [27], there are only 20 ASes (tier-1 providers) which form the dense core of the Internet. These ASes are connected almost as a clique, while the rest of the ASes have far less dense peering connectivity. So when the size of an overlay is reasonably big (e.g., $n>100$ ), the number of customer and peering links that cross-AS paths traverse tends to grow much slower than $O\left(n^{2}\right)$. For example, a joining end host may only add one customer link to the overlay topology, and share the peering links that have been used by other end hosts. Meanwhile, only a few nodes are needed in a single AS before link sharing occurs in paths within an AS.

We believe this heavy sharing accounts for our empirical observation that $k=O(n)$ in a real router-level topology, and $k$ grows at worst like $O(n \log n)$ in several generated topologies. Note that the real 284,805-router topology represents a fairly complete transit portion of the Internet [25]. In our analysis, we conservatively assume that there is only one end host connecting to each edge router to reduce the possible path sharing, but we still find $k=O(n)$ when $n>100$.

\section{DYNAMIC ALGORITHMS FOR TOPOL- OGY CHANGES}

During normal operation, new links may appear or disappear, routing paths between end hosts may change, and hosts may enter or exit the overlay network. These changes may cause rows or columns to be added to or removed from $G$, or entries in $G$ may change. In this section, we design efficient algorithms to incrementally adapt to these changes.

\subsection{Path Additions and Deletions}

The basic building blocks for topology updates are path additions and deletions. We have already handled path additions in Algorithm 1; adding a path $v$ during an update is no different than adding a path $v$ during the initial scan of $G$. In both cases, we decide whether to add $v$ to $\bar{G}$ and update $R$.

To delete a path that is not in $\bar{G}$ is trivial; we just remove it from $G$. But to remove a path from $\bar{G}$ is more complicated. We need to update $R$; this can be done in $O\left(k^{2}\right)$ time by standard algorithms (see e.g. Algorithm 3.4 in [28, p.338]). In general, we may then need to replace the deleted path with another measurement path. Finding a replacement path, or deciding that no such path is needed, can be done by re-scanning the rows of $G$ as in Algorithm 1; however, this would take time $O\left(r k^{2}\right)$.

We now describe Algorithm 2 to delete a path $v$ more efficiently. Suppose $v$ corresponds to the $i$ th row in $\bar{G}$ and the $j$ th row in $G$, we define $\bar{G}^{\prime} \in \mathbb{R}^{(k-1) \times s}$ as the measurement path matrix after deleting the $i$ th row, and $G^{\prime} \in \mathbb{R}^{(r-1) \times s}$ as the path matrix after removing the $j$ th row. By deleting 


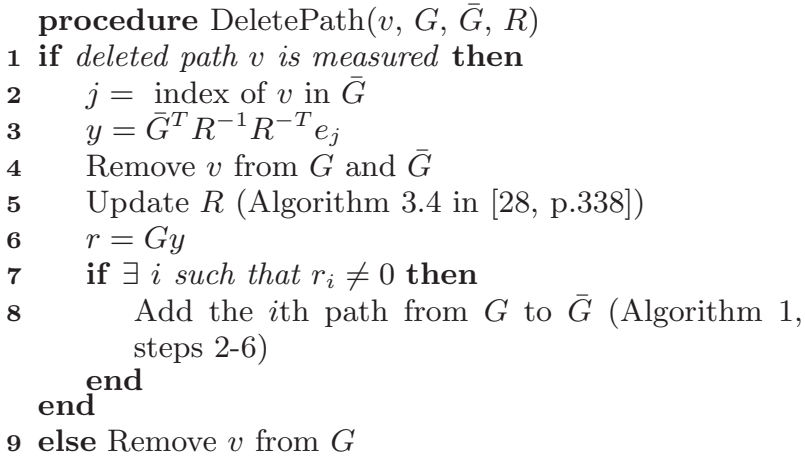

Algorithm 2: Path deletion algorithm

$v$ from $\bar{G}$, we reduce the dimension of $\bar{G}$ from $k$ to $k-1$. Intuitively, our algorithm works in the following two steps.

1. Find a vector $y$ that only describes the direction removed by deleting the $i$ th row of $\bar{G}$.

2. Test if the path space of $G^{\prime}$ is orthogonal to that direction, i.e., find whether there is any path $p \in G^{\prime}$ that has a non-zero component on that direction. If not, no replacement path is needed. Otherwise, replace $v$ with any of such path $p$, and update the QR decomposition.

Next, we describe how each step is implemented. To find $y$ which is in the path space of $\bar{G}$ but not of $\bar{G}^{\prime}$, we solve the linear system $\bar{G} y=e_{i}$, where $e_{i}$ is the vector of all zeros except for a one in entry $i$. This system is similar to the linear system we solved to find $x_{G}$, and one solution is $y=\bar{G}^{T} R^{-1} R^{-T} e_{i}$.

Once we have computed $y$, we compute $r=G^{\prime} y$, where $G^{\prime}$ is the updated $G$ matrix. Because we chose $y$ to make $\bar{G}^{\prime} y=0$, all the elements of $r$ corresponding to selected rows are zero. Paths such that $r_{j} \neq 0$ are guaranteed to be independent of $\bar{G}^{\prime}$, since if row $j$ of $G$ could be written as $w^{T} \bar{G}^{\prime}$ for some $w$, then $r_{j}$ would be $w^{T} \bar{G}^{\prime} y=0$. If all elements of $r$ are zero, then $y$ is a null vector for all of $G^{\prime}$; in this case, the dimension $k^{\prime}$ of the row space of $G^{\prime}$ is $k-1$, and we do not need to replace the deleted measurement path. Otherwise, we can find any $j$ such that $r_{j} \neq 0$ and add the $j$ th path to $\bar{G}^{\prime}$ to replace the deleted path.

Take the overlay network in Fig. 3 for example, suppose $\bar{G}$ is composed of the paths $A B$ and $B C$, i.e., $\bar{G}=\left[\begin{array}{lll}1 & 1 & 0 \\ 1 & 1 & 1\end{array}\right]$. Then we delete path $B C, \bar{G}^{\prime}=\left[\begin{array}{lll}1 & 1 & 0\end{array}\right]^{T}$ and $G^{\prime}=\left[\begin{array}{lll}1 & 1 & 0 \\ 0 & 0 & 1\end{array}\right]$. Applying Algorithm 2, we have $y=\left[\begin{array}{lll}0 & 0 & 1\end{array}\right]^{T}$ and $r=$ $\left[\begin{array}{ll}0 & 1\end{array}\right]^{T}$. Thus the second path in $G^{\prime}, A C$, should be added to $\bar{G}^{\prime}$. If we visualize such path deletion in reference to the geometry of the linear system, the path space of $G^{\prime}$ remains as a plane in Fig. 3, but $\bar{G}^{\prime}$ only has one dimension of the path space left, so we need to add $A C$ to $\bar{G}^{\prime}$.

When deleting a path used in $\bar{G}$, the factor $R$ can be updated in $O\left(k^{2}\right)$ time. To find a replacement row, we need to compute a sparse matrix-vector product involving $G$, which takes $O\left(n^{2} \times\right.$ (average path length) $)$ operations. Since most routing paths are short, the dominant cost will typically be the update of $R$. Therefore, the complexity of Algorithm 2 is $O\left(k^{2}\right)$.

\subsection{End Hosts Join/Leave the Overlay}

To add an end host $h$, we use Algorithm 1 to scan all the new paths from $h$, for a cost of $O\left(n k^{2}\right)$. However, it is inefficient to delete an end host $h$ by directly using Algorithm 2 to delete all affected paths. If Algorithm 2 is used to delete a path that starts/ends at $h$, often another path that starts/ends at $h$ is chosen as a replacement - and soon deleted in turn. To avoid this behavior, we remove all these paths from $G$ first, then use the updated $G$ in Algorithm 2 to select replacements as needed during the removal of paths that start/end at $h$. Each path in $\bar{G}$ can be removed in $O\left(k^{2}\right)$ time; the worst-case total cost of end host deletion is then $O\left(n k^{2}\right)$.

\subsection{Routing Changes}

In the network, routing changes or link failures can affect multiple paths in $G$. Previous studies have shown that end-to-end Internet paths generally tend to be stable for significant lengths of time, e.g., for at least a day [29, 30]. So we can incrementally measure the topology to detect changes. Each end host measures the paths to all other end hosts daily, and for each end host, such measurement load can be evenly distributed throughout the day. In addition to the periodic route measurement, if any path is found to have large loss rate changes, we will check its route instantly.

For each link, we keep a list of the paths that traverse it. If any path is reported as changed for certain $\operatorname{link}(\mathrm{s})$, we will examine all other paths that go through those link(s) because it is highly likely that those paths can change their routes as well. We use Algorithms 1 and 2 to incrementally incorporate each path change.

Unlike $O\left(n^{2}\right)$ approaches (e.g., RON), we need some extra traceroute measurement. However, the key point is that the end-to-end routing remains much more stable than its loss rate, thus requires far less frequent measurement. So the savings on loss rate probing dwarf the traceroute overhead.

\section{LOAD BALANCING AND TOPOLOGY ERROR HANDLING}

To further improve the scalability and accuracy, we need to have good load balancing and handle topology measurement errors, as discussed in this section.

\subsection{Measurement Load Balancing}

To avoid overloading any single node or its access link, we evenly distribute the measurements among the end hosts. We randomly reorder the paths in $G$ before scanning them for selection in Algorithm 1. Since each path has equal probability of being selected for monitoring, the measurement load on each end host is similar. Note any basis set generated from Algorithm 1 is sufficient to describe all paths $G$. Thus the load balancing has no effect on the loss rate estimation accuracy.

\subsection{Handling Topology Measurement Errors}

As our goal is to estimate the end-to-end path loss rate instead of any interior link loss rate, we can tolerate certain topology measurement inaccuracies, such as incomplete routing information and poor router alias resolution.

For completely untraceable paths, we add a direct link between the source and the destination. In our system, these paths will become selected paths for monitoring. For paths with incomplete routing information, we add links 
from where the normal route becomes unavailable (e.g., self loops or displaying "***" in traceroute), to where the normal route resumes or to the destination if such anomalies persist until the end. For instance, if the measured route is $\left(s r c, i p_{1}, " * * * *, i p_{2}\right.$, dest), the path is composed of three links: $\left(\operatorname{src} i p_{1}\right),\left(i p_{1}, i p_{2}\right)$, and $\left(i p_{2}, d e s t\right)$. By treating the untraceable path (segment) as a normal link, the resulting topology is equivalent to the one with complete routing information for calculating the path loss rates.

For topologies with router aliases presenting one physical link as several links, we have little need to resolve these aliases. At worst, our failure to recognize the links as the same will result in a few more path measurements because the rank of $G$ will be higher. For these links, their corresponding entries in $x_{G}$ will be assigned similar values because they are actually a single link. Thus the path loss rate estimation accuracy is not affected, as verified by Internet experiments in Sec. 8. In addition, our system is robust to measurement node failures and node changes by providing bounds on the estimated loss rates.

\section{EVALUATION}

In this section, we present our evaluation metrics, simulation methodology and simulation results.

\subsection{Metrics}

The metrics include path loss rate estimation accuracy, variation of measurement loads among the end hosts, and speed of setup, update, and topology change adaptation.

To compare the inferred loss rate $\hat{p}$ with real loss rate $p$, we analyze both absolute error and error factor. The absolute error is $|p-\hat{p}|$. We adopt the error factor $F_{\varepsilon}(p, \hat{p})$ defined in [9] as follows:

$$
F_{\varepsilon}(p, \hat{p})=\max \left\{\frac{p(\varepsilon)}{\hat{p}(\varepsilon)}, \frac{\hat{p}(\varepsilon)}{p(\varepsilon)}\right\}
$$

where $p(\varepsilon)=\max (\varepsilon, p)$ and $\hat{p}(\varepsilon)=\max (\varepsilon, \hat{p})$. Thus, $p$ and $\hat{p}$ are treated as no less than $\varepsilon$, and then the error factor is the maximum ratio, upwards or downwards, by which they differ. We use the default value $\varepsilon=0.001$ as in [9]. If the estimation is perfect, the error factor is one.

Furthermore, we classify a path to be lossy if its loss rate exceeds $5 \%$, which is the threshold between "tolerable loss" and "serious loss" as defined in [20]. We report the true number of lossy paths, the percentage of real lossy paths identified (coverage) and the false positive rate, all averaged over five runs of experiment for each configuration.

There are two types of measurement load: 1) sending probes, and 2) receiving probes and computing loss rates. The load reflects the CPU and uplink/downlink bandwidth consumption. For each end host $h$, its measurement load is linearly proportional to, and thus denoted by the number of monitored paths with $h$ as sender/receiver. Then we compute its variation across end hosts in terms of the coefficient of variation (CV) and the maximum vs. mean ratio (MMR), for sending load and receiving load separately. The CV of a distribution $x$, defined as below, is a standard metric for measuring inequality of $x$, while the MMR checks if there is any single node whose load is significantly higher than the average load.

$$
C V(x)=\frac{\text { standard deviation }(x)}{\text { mean }(x)}
$$

The simulations only consider undirected links, so for each monitored path, we randomly select one end host as sender and the other as receiver. This is applied to all simulations with or without load balancing.

\subsection{Simulation Methodology}

We consider the following dimensions for simulation.

- Topology type: three types of synthetic topologies from BRITE (see Sec. 7.3) and a real router-level topology from [25]. All the hierarchical models have similar results, we just use Barabasi-Albert at the AS level and Waxman at the router level as the representative.

- Topology size: the number of nodes ranges from 1000 to $2000{ }^{2}$. Note that the node count includes both internal nodes (i.e., routers) and end hosts.

- Fraction of end hosts on the overlay network: we define end hosts to be the nodes with the least degree. Then we randomly choose from $10 \%$ to $50 \%$ of end hosts to be on the overlay network. This gives us pessimistic results because other distributions of end hosts will probably have more sharing of the routing paths among them. We prune the graphs to remove the nodes and links that are not referenced by any path on the overlay network.

- Link loss rate distribution: $90 \%$ of the links are classified as "good" and the rest as "bad". We use two different models for assigning loss rate to links as in [10]. In the first model $\left(L L R D_{1}\right)$, the loss rate for good links is selected uniformly at random in the $0-1 \%$ range and that for bad links is chosen in the $5-10 \%$ range. In the second model $\left(L L R D_{2}\right)$, the loss rate ranges for good and bad links are $0-1 \%$ and $1-100 \%$ respectively. Given space limitations, most results are under model $L L R D_{1}$ except for Sec. 7.4.

- Loss model: After assigning each link a loss rate, we use either a Bernoulli or a Gilbert model to simulate the loss processes at each link. For a Bernoulli model, each packet traversing a link is dropped at independently fixed probability as the loss rate of the link. For a Gilbert model, the link fluctuates between a good state (no packet dropped) and a bad state (all packets dropped). According to Paxon's observed measurement of Internet [31], the probability of remaining in bad state is set to be $35 \%$ as in [10]. Thus, the Gilbert model is more likely to generate bursty losses than the Bernoulli model. The other state transition probabilities are selected so that the average loss rates matches the loss rate assigned to the link.

We repeat our experiments five times for each simulation configuration unless denoted otherwise, where each repetition has a new topology and new loss rate assignments. The path loss rate is simulated based on the transmission of 10000 packets. Using the loss rates of selected paths as input, we compute $x_{G}$, then the loss rates of all other paths.

\footnotetext{
${ }^{2} 20000$ is the largest topology we can simulate on a $1.5 \mathrm{GHz}$ Pentium 4 machine with 512M memory.
} 

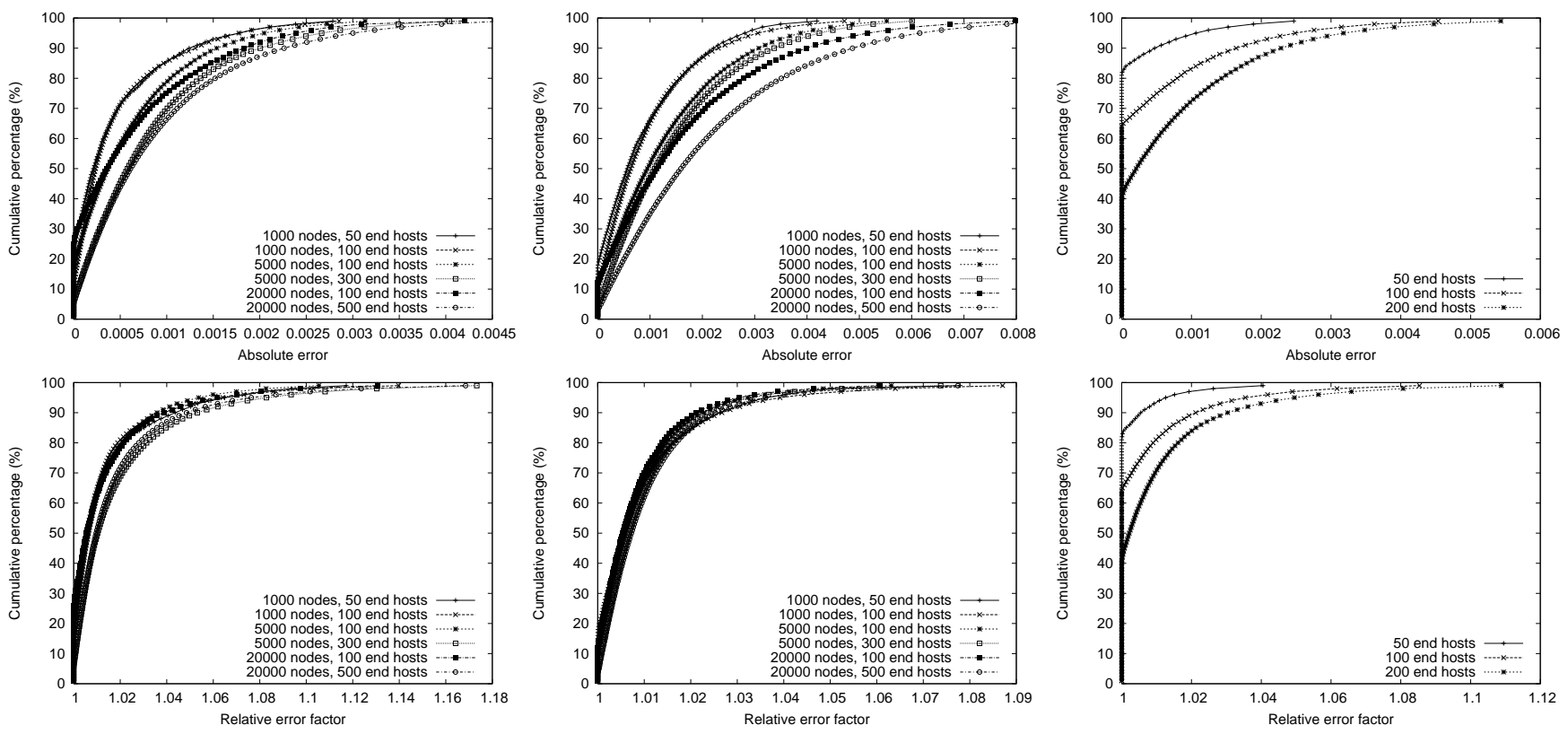

BRITE Barabasi-Albert topology

BRITE Hierarchical topology

Real topology of $284 \mathrm{~K}$ routers

Figure 5: Cumulative distribution of absolute errors (top) and error factors (bottom) under Gilbert loss model for various topologies.

\begin{tabular}{|c|c|c|c|c|c|c|c|c|c|c|c|c|c|}
\hline \multirow{2}{*}{$\begin{array}{c}\# \text { of } \\
\text { nodes }\end{array}$} & \multicolumn{2}{|c|}{ \# of end hosts } & \multirow{2}{*}{$\begin{array}{c}\text { \# of } \\
\text { paths }(r)\end{array}$} & \multicolumn{2}{|c|}{ \# of links } & \multirow{2}{*}{$\begin{array}{c}\operatorname{rank} \\
(k)\end{array}$} & \multirow{2}{*}{$\begin{array}{l}\text { MPR } \\
(k / r)\end{array}$} & \multicolumn{3}{|c|}{ lossy paths (Bernoulli) } & \multicolumn{3}{|c|}{ lossy paths (Gilbert) } \\
\hline & total & $\mathrm{OL}(n)$ & & original & $\overline{\mathrm{AP}}$ & & & real & coverage & $\mathrm{FP}$ & real & coverage & $\mathrm{FP}$ \\
\hline \multirow{2}{*}{1000} & \multirow{2}{*}{506} & 50 & 1225 & \multirow{2}{*}{1997} & 443 & 275 & $22 \%$ & 437 & $99.6 \%$ & $1.3 \%$ & 437 & $100.0 \%$ & $0.2 \%$ \\
\hline & & 100 & 4950 & & 791 & 543 & $11 \%$ & 2073 & $99.0 \%$ & $2.0 \%$ & 1688 & $99.9 \%$ & $0.2 \%$ \\
\hline \multirow{2}{*}{5000} & \multirow{2}{*}{2489} & 100 & 4950 & \multirow{2}{*}{9997} & 1615 & $\overline{929}$ & $19 \%$ & 2271 & $99.1 \%$ & $2.0 \%$ & 2277 & $99.7 \%$ & $0.1 \%$ \\
\hline & & 300 & 44850 & & 3797 & 2541 & $6 \%$ & 19952 & $98.6 \%$ & $4.1 \%$ & 20009 & $99.6 \%$ & $0.3 \%$ \\
\hline \multirow{2}{*}{20000} & \multirow{2}{*}{10003} & 100 & 4950 & \multirow{2}{*}{39997} & 2613 & 1318 & $27 \%$ & 2738 & $98.4 \%$ & $3.4 \%$ & 2446 & $99.5 \%$ & $0.6 \%$ \\
\hline & & 500 & 124750 & & 11245 & 6755 & $5 \%$ & 67810 & $97.8 \%$ & $5.5 \%$ & 64733 & $99.5 \%$ & $0.4 \%$ \\
\hline
\end{tabular}

\begin{tabular}{|c|c|c|c|c|c|c|c|c|c|c|c|c|c|}
\hline $\begin{array}{c}\text { \# of } \\
\text { nodes }\end{array}$ & \multicolumn{2}{|c|}{ \# of end hosts } & $\begin{array}{c}\text { \# of } \\
\text { paths }(r)\end{array}$ & \multicolumn{2}{|c|}{ \# of links } & $\begin{array}{c}\text { rank } \\
(k)\end{array}$ & $\begin{array}{l}\text { MPR } \\
(k / r)\end{array}$ & \multicolumn{3}{|c|}{ lossy paths (Bernoulli) } & \multicolumn{3}{|c|}{ lossy paths (Gilbert) } \\
\hline 1000 & 335 & 100 & 4950 & 2000 & 1238 & 909 & $18 \%$ & 2544 & $98.5 \%$ & $4.6 \%$ & 2539 & $99.7 \%$ & $0.5 \%$ \\
\hline 5000 & 1680 & 300 & 44850 & 10000 & 6263 & 4563 & $10 \%$ & 29135 & $96.8 \%$ & $7.1 \%$ & 28782 & $99.1 \%$ & $1.1 \%$ \\
\hline \multirow{2}{*}{20000} & \multirow{2}{*}{6750} & 100 & 4950 & \multirow{2}{*}{40000} & 5438 & 2606 & $53 \%$ & 3735 & $98.4 \%$ & $2.3 \%$ & 3607 & $99.6 \%$ & $0.4 \%$ \\
\hline & & 500 & 124750 & & 20621 & 13769 & $11 \%$ & 93049 & $96.1 \%$ & $5.7 \%$ & 92821 & $99.1 \%$ & $1.5 \%$ \\
\hline
\end{tabular}

\begin{tabular}{|c|c|c|c|c|c|c|c|c|c|c|c|c|c|}
\hline \multirow{2}{*}{$\begin{array}{c}\text { \# of } \\
\text { nodes }\end{array}$} & \multicolumn{2}{|c|}{ \# of end hosts } & \multirow{2}{*}{$\begin{array}{c}\text { \# of } \\
\text { paths }(r)\end{array}$} & \multicolumn{2}{|c|}{ \# of links } & \multirow{2}{*}{$\begin{array}{c}\operatorname{rank} \\
(k)\end{array}$} & \multirow{2}{*}{$\begin{array}{l}\text { MPR } \\
(k / r)\end{array}$} & \multicolumn{3}{|c|}{ lossy paths (Bernoulli) } & \multicolumn{3}{|c|}{ lossy paths (Gilbert) } \\
\hline & total & $\mathrm{OL}(n)$ & & original & $\mathrm{AP}$ & & & real & coverage & $\mathrm{FP}$ & real & coverage & FP \\
\hline 1000 & 312 & 100 & 4950 & 2017 & 796 & 481 & $10 \%$ & 4207 & $98.4 \%$ & $1.6 \%$ & 3979 & $99.6 \%$ & $0.3 \%$ \\
\hline 5000 & 1608 & 300 & 44850 & 10047 & 3076 & 1787 & $4 \%$ & 42331 & $99.2 \%$ & $0.8 \%$ & 42281 & $99.8 \%$ & $0.1 \%$ \\
\hline \multirow{2}{*}{20000} & \multirow{2}{*}{6624} & 100 & 4950 & \multirow{2}{*}{40077} & 2034 & 613 & $12 \%$ & 4847 & $99.8 \%$ & $0.2 \%$ & 4830 & $100.0 \%$ & $0.1 \%$ \\
\hline & & 500 & 124750 & & 7460 & 3595 & $3 \%$ & 122108 & $99.5 \%$ & $0.3 \%$ & 121935 & $99.9 \%$ & $0.1 \%$ \\
\hline
\end{tabular}

\begin{tabular}{|c|c|c|c|c|c|c|c|c|c|c|}
\hline \multirow{2}{*}{$\begin{array}{l}\text { \# of end hosts } \\
\text { on overlay }(n)\end{array}$} & \multirow{2}{*}{$\begin{array}{c}\text { \# of } \\
\text { paths }(r)\end{array}$} & \multirow{2}{*}{$\begin{array}{c}\text { \# of links } \\
\text { after pruning }\end{array}$} & \multirow{2}{*}{$\begin{array}{c}\text { rank } \\
(k)\end{array}$} & \multirow{2}{*}{$\begin{array}{l}\text { MPR } \\
(k / r)\end{array}$} & \multicolumn{3}{|c|}{ lossy paths (Bernoulli) } & \multicolumn{3}{|c|}{ lossy paths (Gilbert) } \\
\hline & & & & & real & coverage & $\overline{\mathrm{FP}}$ & real & coverage & FP \\
\hline 50 & 1225 & 2098 & 1017 & $83 \%$ & 891 & $99.7 \%$ & $0.9 \%$ & 912 & $100.0 \%$ & $0.2 \%$ \\
\hline 100 & 4950 & 5413 & 3193 & $65 \%$ & 3570 & $98.7 \%$ & $1.9 \%$ & 3651 & $99.6 \%$ & $0.3 \%$ \\
\hline 200 & 19900 & 12218 & 8306 & $42 \%$ & 14152 & $97.9 \%$ & $3.1 \%$ & 14493 & $99.6 \%$ & $0.4 \%$ \\
\hline
\end{tabular}

Table 2: Simulation results for three types of BRITE router topologies: Barabasi-Albert (top), Waxman (upper middle) and hierarchical model (lower middle), and a real router topology (bottom). OL gives the number of end hosts on the overlay network. AP shows the number of links after pruning (i.e., remove the nodes and links that are not on the overlay paths). MPR (monitored path ratio) is the fraction of the total end-to-end paths which we monitor. FP is the false positive rate. 


\begin{tabular}{|c|c|c|c|c|c|c|c|}
\hline \multirow{2}{*}{$\begin{array}{c}\text { \# of } \\
\text { nodes }\end{array}$} & \multicolumn{2}{|c|}{ end hosts } & \multicolumn{2}{c|}{ lossy paths (Gilbert) } & \multicolumn{2}{c|}{ speed (second) } \\
\cline { 2 - 8 } & total & OL & real & coverage & FP & setup & update \\
\hline \multirow{2}{*}{1000} & \multirow{2}{*}{506} & 50 & 495 & $99.8 \%$ & $1.1 \%$ & 0.13 & 0.08 \\
\cline { 3 - 8 } & & 100 & 1989 & $99.8 \%$ & $3.0 \%$ & 0.91 & 0.17 \\
\hline \multirow{2}{*}{5000} & \multirow{2}{*}{2489} & 100 & 2367 & $99.6 \%$ & $3.5 \%$ & 1.98 & 0.22 \\
\cline { 3 - 8 } & & 300 & 21696 & $99.2 \%$ & $1.4 \%$ & 79.0 & 1.89 \\
\hline \multirow{2}{*}{20000} & \multirow{2}{*}{10003} & 100 & 2686 & $98.8 \%$ & $1.1 \%$ & 3.00 & 0.25 \\
\cline { 3 - 8 } & & 500 & 67817 & $99.0 \%$ & $4.6 \%$ & 1250 & 4.33 \\
\hline
\end{tabular}

Table 3: Simulation results with model $L L R D_{2}$. Use the same Barabasi-Albert topologies as in Table 7 . Refer to Table 7 for statistics like rank. FP is the false positive rate. OL means overlay network.

\subsection{Results for Different Topologies}

For all topologies in Sec. 7.2, we achieve high loss rate estimation accuracy. Results for the Bernoulli and the Gilbert models are similar. Since the Gilbert loss model is more realistic, we plot the cumulative distribution functions (CDFs) of absolute errors and error factors with the Gilbert model in Fig. 5. For all the configurations, the absolute errors are less than 0.008 and the error factors are less than 1.18. Waxman topologies have similar results, and we omit them in the interest of space.

The lossy path inference results are shown in Table 7. Notice that $k$ is much smaller than the number of IP links that the overlay network spans, which means that there are many IP links whose loss rates are unidentifiable. Although different topologies have similar asymptotic regression trend for $k$ as $O(n \log n)$, they have different constants. For an overlay network with given number of end hosts, the more IP links it spans on, the bigger $k$ is. We found that Waxman topologies have the largest $k$ among all synthetic topologies. For all configurations, the lossy path coverage is more than $96 \%$ and the false positive ratio is less than $8 \%$. Many of the false positives and false negatives are caused by small estimation errors for paths with loss rates near the $5 \%$ threshold.

We also test our algorithms in the 284,805 -node real routerlevel topology from [25]. There are 65,801 end host routers and 860,683 links. We get the same trend of results as illustrated in Fig. 5 and Table 7. The CDFs include all the path estimates, including the monitored paths for which we know the real loss rates. Given the same number of end hosts, the ranks in the real topology are higher than those of the synthetic ones. But as we find in Sec. 4, the growth of $k$ is still bounded by $O(n)$.

\subsection{Results for Different Link Loss Rate Dis- tribution and Running Time}

We have also run all the simulations above with model $L L R D_{2}$. The loss rate estimation is a bit less accurate than it is under $L L R D_{1}$, but we still find over $95 \%$ of the lossy paths with a false positive rate under $10 \%$. Given space limitations, we only show the lossy path inference with the Barabasi-Albert topology model and the Gilbert loss model in Table 3.

The running time for $L L R D_{1}$ and $L L R D_{2}$ are similar, as in Table 3. All speed results in this paper are based on a $1.5 \mathrm{GHz}$ Pentium 4 machine with 512M memory. Note that it takes about 20 minutes to setup (select the measurement paths) for an overlay of 500 end hosts, but only several seconds for an overlay of size 100. The update (loss rate calculation) time is small for all cases, only 4.3 seconds for 124,750 paths. Thus it is feasible to update online.

\subsection{Results for Measurement Load Balancing}

We examine the measurement load distribution for both synthetic and real topologies, and the results are shown in Table 4. Given the space constraints, we only show the results for Barabasi-Albert and hierarchical model. Our load balancing scheme reduces $\mathrm{CV}$ and MMR substantially for all cases, and especially for MMR. For instance, a 500-node overlay on a 20000-node network of Barabasi-Albert model has its MMR reduced by 7.3 times.

We further plot the histogram of measurement load distribution by putting the load values of each node into 10 equally spaced bins, and counting the number of nodes in each bin as $y$-axis. The $x$-axis denotes the center of each bin, as illustrated in Fig. 6. With load balancing, the histogram roughly follow the normal distribution. In contrast, the histogram without load balancing is close to an exponential distribution. Note that the y-axis in this plot is logarithmic: an empty bar means that the bin contains one member, and 0.1 means the bin is empty.

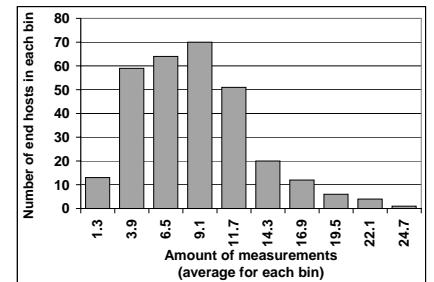

(a) with load balancing

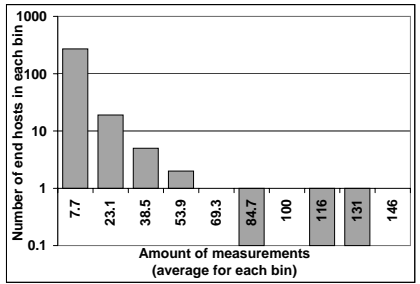

(b) without load balancing
Figure 6: Histogram of the measurement load distribution (as sender) for an overlay of 300 end hosts on a 5000-node Barabasi-Albert topology.

\subsection{Results for Topology Changes}

We study two common scenarios in P2P and overlay networks: end hosts joining and leaving as well as routing changes. Again, the Bernoulli and the Gilbert models have similar results, thus we only show those of the Gilbert model.

\subsubsection{End hosts join/leave}

\begin{tabular}{|c|c|c|c|c|c|}
\hline \# of end & \multirow{2}{*}{$\begin{array}{c}\text { \# of } \\
\text { hosts }\end{array}$} & rank & \multicolumn{3}{|c|}{ lossy paths } \\
\cline { 4 - 6 } & & & real & coverage & FP \\
\hline 40 & 780 & 616 & 470 & $99.9 \%$ & $0.2 \%$ \\
\hline+5 & +210 & +221 & +153 & $100.0 \%$ & $0.1 \%$ \\
$(45)$ & $(990)$ & $(837)$ & $(623)$ & & \\
\hline+5 & +235 & +160 & +172 & $99.8 \%$ & $0.2 \%$ \\
$(50)$ & $(1225)$ & $(997)$ & $(795)$ & & \\
\hline
\end{tabular}

Table 5: Simulation results for adding end hosts on a real router topology. $\mathrm{FP}$ is the false positive rate. Denoted as "+added_value (total_value)".

\begin{tabular}{|c|c|c|c|c|c|}
\hline \# of end & \# of & rank & \multicolumn{3}{|c|}{ lossy paths } \\
\cline { 4 - 6 } hosts & paths & & real & coverage & FP \\
\hline 60 & 1770 & 1397.0 & 1180.3 & $99.9 \%$ & $0.2 \%$ \\
\hline-5 & -285 & -245.3 & -210.0 & $99.8 \%$ & $0.2 \%$ \\
$(55)$ & $(1485)$ & $(1151.7)$ & $(970.3)$ & & \\
\hline-10 & -260 & -156.7 & -150.6 & $99.9 \%$ & $0.1 \%$ \\
$(50)$ & $(1225)$ & $(995.0)$ & $(819.7)$ & & \\
\hline
\end{tabular}

Table 6: Simulation results for deleting end hosts on a real router topology. FP is the false positive rate. Denoted as "-reduced_value (total_value)". 


\begin{tabular}{|c|c|c|c|c|c|c|c|c|c|c|c|c|c|c|c|c|c|}
\hline \multirow{4}{*}{$\begin{array}{c}\text { \# of } \\
\text { nodes }\end{array}$} & \multirow{4}{*}{$\begin{array}{c}\text { OL } \\
\text { size } \\
(n)\end{array}$} & \multicolumn{8}{|c|}{ Barabasi-Albert model } & \multicolumn{8}{|c|}{ hierarchical model } \\
\hline & & \multicolumn{4}{|c|}{ receiver } & \multicolumn{4}{|c|}{ MMR } & \multicolumn{4}{|c|}{$\mathrm{CV}$} & \multicolumn{4}{|c|}{ MMR } \\
\hline & & \multicolumn{2}{|c|}{ sender } & \multicolumn{2}{|c|}{ receiver } & \multicolumn{2}{|c|}{ sender } & \multicolumn{2}{|c|}{ receiver } & \multicolumn{2}{|c|}{ sender } & \multicolumn{2}{|c|}{ receiver } & \multicolumn{2}{|c|}{ sender } & \multicolumn{2}{|c|}{ receiver } \\
\hline & & $\mathrm{LB}$ & NLB & LB & $\mathrm{NLB}$ & $\mathrm{LB}$ & NLB & $\mathrm{LB}$ & NLB & LB & NLB & $\mathrm{LB}$ & $\mathrm{NLB}$ & $\mathrm{LB}$ & NLB & $\mathrm{LB}$ & NLB \\
\hline \multirow{2}{*}{1000} & 50 & 0.62 & 1.10 & 0.56 & 0.94 & 2.41 & 5.91 & 3.07 & 4.09 & 0.52 & 0.96 & 0.53 & 0.87 & 2.28 & 4.80 & 2.51 & 4.29 \\
\hline & 100 & 0.61 & 1.42 & 0.64 & 1.34 & 3.21 & 11.33 & 3.61 & 10.67 & 0.51 & 1.38 & 0.47 & 1.39 & 2.74 & 10.06 & 2.32 & 10.27 \\
\hline \multirow[b]{2}{*}{5000} & 100 & 0.44 & 0.89 & 0.47 & 0.97 & 2.25 & 6.11 & 2.36 & 6.50 & 0.49 & 1.18 & 0.53 & 1.39 & 2.60 & 9.18 & 2.97 & 10.16 \\
\hline & 300 & 0.52 & 1.59 & 0.51 & 1.51 & 2.97 & 18.70 & 2.74 & 17.25 & 0.47 & 1.72 & 0.48 & 1.76 & 3.47 & 23.93 & 4.13 & 25.76 \\
\hline \multirow{2}{*}{20000} & 100 & 0.36 & 0.55 & 0.40 & 0.59 & 1.93 & 3.20 & 2.29 & 3.69 & 0.48 & 1.17 & 0.43 & 1.09 & 3.04 & 8.86 & 2.56 & 7.09 \\
\hline & 500 & 0.52 & 1.36 & 0.53 & 1.35 & 2.64 & 19.21 & 3.01 & 16.82 & 0.46 & 1.85 & 0.46 & 1.89 & 5.01 & 25.85 & 5.56 & 27.67 \\
\hline
\end{tabular}

Table 4: Measurement load (as sender or receiver) distribution for various BRITE topologies. OL Size is the number of end hosts on overlay. "LB" means with load balancing, and "NLB" means without load balancing.

For the real router topology, we start with an overlay network of 40 random end hosts. Then we randomly add an end host to join the overlay, and repeat the process until the size of the overlay reaches 45 and 50. Averaged over three runs, the results in Table 5 show that there is no obvious accuracy degradation caused by accumulated numerical errors. The average running time for adding a path is $125 \mathrm{msec}$, and for adding a node, 1.18 second. Notice that we add a block of paths together to speedup adding node (Sec. 3.2).

Similarly, for removing end hosts, we start with an overlay network of 60 random end hosts, then randomly select an end host to delete from the overlay, and repeat the process until the size of the overlay is reduced to 55 and 50. Again, the accumulated numerical error is negligible as shown in Table 6. As shown in Sec. 5 , deleting a path in $\bar{G}$ is much more complicated than adding a path. With the same machine, the average time for deleting a path is $445 \mathrm{msec}$, and for deleting a node, 16.9 seconds. We note that the current implementation is not optimized: we can speed up node deletion by processing several paths simultaneously, and we can speed up path addition and deletion with iterative methods such as CGNE or GMRES [32]. Since the time to add/delete a path is $O\left(k^{2}\right)$, and to add/delete a node is $O\left(n k^{2}\right)$, we expect our updating scheme to be substantially faster than the $O\left(n^{2} k^{2}\right)$ cost of re-initialization for larger $n$.

\subsubsection{Routing changes}

We form an overlay network with 50 random end hosts on the real router topology. Then we simulate topology changes by randomly choosing a link that is on some path of the overlay and removing of such a link will not cause disconnection for any pair of overlay end hosts. Then we assume that the link is broken, and re-route the affected path(s). Algorithms in Sec. 5 incrementally incorporate each path change. Averaged over three runs, results in Table 7 show that we adapt quickly, and still have accurate path loss rate estimation.

\begin{tabular}{|c|c|}
\hline \# of paths affected & 40.7 \\
\hline \# of monitored paths affected & 36.3 \\
\hline \# of unique nodes affected & 41.7 \\
\hline \# of real lossy paths (before/after) & $761.0 / 784.0$ \\
\hline coverage (before/after) & $99.8 \% / 99.8 \%$ \\
\hline false positive rate (before/after) & $0.2 \% / 0.1 \%$ \\
\hline average running time & 17.3 seconds \\
\hline
\end{tabular}

Table 7: Simulation results for removing a link from a real router topology.

We also simulate the topology changes by adding a random link on some path(s) of the overlay. The results are similar as above, so we omit them here for brevity.

\section{INTERNET EXPERIMENTS}

We implemented our system on the PlanetLab [33] testbed. In this section, we present the implementation results.

\subsection{Methodology}

We choose 51 PlanetLab hosts, each from a different organization as shown in Table 8 . All the international PlanetLab hosts are universities.

\begin{tabular}{|c|c|c|c|}
\hline \multicolumn{3}{|c|}{ Areas and Domains } & \# of hosts \\
\hline \multirow{5}{*}{ US (40) } & \multicolumn{2}{|c|}{.$e d u$} & 33 \\
\hline & \multicolumn{2}{|c|}{.org } & 3 \\
\hline & \multicolumn{2}{|c|}{.net } & 2 \\
\hline & \multicolumn{2}{|c|}{.gov } & 1 \\
\hline & \multicolumn{2}{|c|}{.$u s$} & 1 \\
\hline \multirow{9}{*}{$\begin{array}{l}\text { Inter- } \\
\text { national } \\
(11)\end{array}$} & \multirow{5}{*}{$\begin{array}{l}\text { Europe } \\
(6)\end{array}$} & France & 1 \\
\hline & & Sweden & 1 \\
\hline & & Denmark & 1 \\
\hline & & Germany & 1 \\
\hline & & UK & 2 \\
\hline & \multirow{2}{*}{$\begin{array}{l}\text { Asia } \\
(2)\end{array}$} & Taiwan & 1 \\
\hline & & Hong Kong & 1 \\
\hline & \multicolumn{2}{|c|}{ Canada } & 2 \\
\hline & \multicolumn{2}{|c|}{ Australia } & 1 \\
\hline
\end{tabular}

Table 8: Distribution of selected PlanetLab hosts.

First, we measure the topology among these sites by simultaneously running "traceroute" to find the paths from each host to all others. Each host saves its destination IP addresses for sending measurement packets later. Then we measure the loss rates between every pair of hosts. Our measurement consists of 300 trials, each of which lasts $300 \mathrm{msec}$. During a trial, each host sends a 40 -byte UDP packet ${ }^{3}$ to every other host. Usually the hosts will finish sending before the $300 \mathrm{msec}$ trial is finished. For each path, the receiver counts the number of packets received out of 300 to calculate the loss rate.

To prevent any host from receiving too many packets simultaneously, each host sends packets to other hosts in a different random order. Furthermore, any single host uses a different permutation in each trial so that each destination has equal opportunity to be sent later in each trial. This is because when sending packets in a batch, the packets sent later are more likely to be dropped. Such random permutations are pre-generated by each host. To ensure that all hosts in the network take measurements at the same time, we set up sender and receiver daemons, then use a wellconnected server to broadcast a "START" command.

Will the probing traffic itself cause losses? We performed sensitivity analysis on sending frequency as shown in Fig. 7.

\footnotetext{
${ }^{3} 20$-byte IP header +8 -byte UDP header +12 -byte data on sequence number and sending time.
} 
All experiments were executed between 1am-3am PDT June 24, 2003, when most networks are free. The traffic rate from or to each host is $(51-1) \times$ sending_freq $\times 40$ bytes $/ \mathrm{sec}$. The number of lossy paths does not change much when the sending rate varies, except when the sending rate is over $12.8 \mathrm{Mbps}$, since many servers can not sustain that sending rate. We choose a $300 \mathrm{msec}$ sending interval to balance quick loss rate statistics collection with moderate bandwidth consumption.

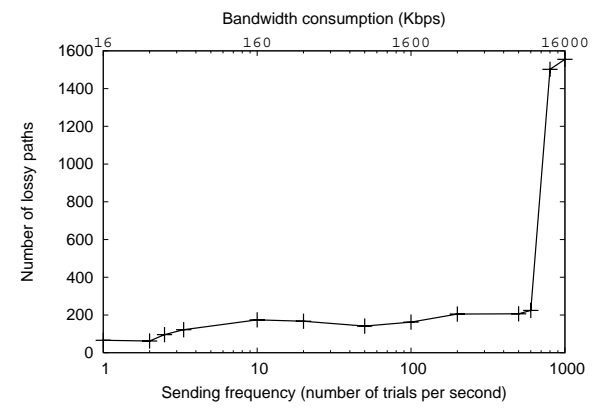

Figure 7: Sensitivity test of sending frequency

Note that the experiments above use $O\left(n^{2}\right)$ measurements so that we can compare the real loss rates with our inferred loss rates. In fact, our technique only requires $O(n \log n)$ measurements. Thus, given good load balancing, each host only needs to send to $O(\log n)$ hosts. In fact, we achieve similar CV and MMR for measurement load distribution as in the simulation. Even for an overlay network of 400 end hosts on the 284K-node real topology used before, $k$ $=18668$. If we reduce the measurement frequency to one trial per second, the traffic consumption for each host is $18668 / 400 \times 40$ bytes $/ \mathrm{sec}=14.9 \mathrm{Kbps}$, which is typically less than $5 \%$ of the bandwidth of today's "broadband" Internet links. We can use adaptive measurement techniques in [3] to further reduce the overheads.

\begin{tabular}{|c|c|c|c|c|c|c|}
\hline \multirow{2}{*}{$\begin{array}{l}\text { loss } \\
\text { rate }\end{array}$} & \multirow{2}{*}{$\begin{array}{l}0, \\
0.05\end{array}$} & \multicolumn{5}{|c|}{ lossy path $[0.05,1.0](4.1 \%)$} \\
\hline & & $0.05,0.1)$ & $0.1,0.3)$ & $0.3,0.5)$ & $0.5,1.0)$ & 1.0 \\
\hline$\%$ & $95.9 \%$ & $15.2 \%$ & $31.0 \%$ & $23.9 \%$ & $4.3 \%$ & $25.6 \%$ \\
\hline
\end{tabular}

Table 9: Loss rate distribution: lossy vs. non-lossy and the sub-percentage of lossy paths.

\subsection{Results}

From June 24 to June 27, 2003, we ran the experiments 100 times, mostly during peak hours 9am - 6pm PDT. Each experiment generates $51 \times 50 \times 300=765 \mathrm{~K}$ UDP packets, totaling $76.5 \mathrm{M}$ packets for all experiments. We run the loss rate measurements three to four times every hour, and run the pair-wise traceroute every two hours. Across the 100 runs, the average number of selected monitoring paths $(\bar{G})$ is 871.9 , about one third of total number of end-to-end paths, 2550. Table 9 shows the loss rate distribution on all the paths of the 100 runs. About $96 \%$ of the paths are nonlossy. Among the lossy paths, most of the loss rates are less than 0.5. Though we try to choose stable nodes for experiments, about $25 \%$ of the lossy paths have $100 \%$ losses and are likely caused by node failures or other reachability problems as discussed in Sec. 8.2.2.

\subsubsection{Accuracy and speed}

When identifying the lossy paths (loss rates $>0.05$ ), the average coverage is $95.6 \%$ and the average false positive rate is $2.75 \%$. Fig. 8 shows the CDFs for the coverage and the false positive rate. Notice that 40 runs have $100 \%$ coverage and 90 runs have coverage over $85 \%$. 58 runs have no false positives and 90 runs have false positive rates less than $10 \%$.

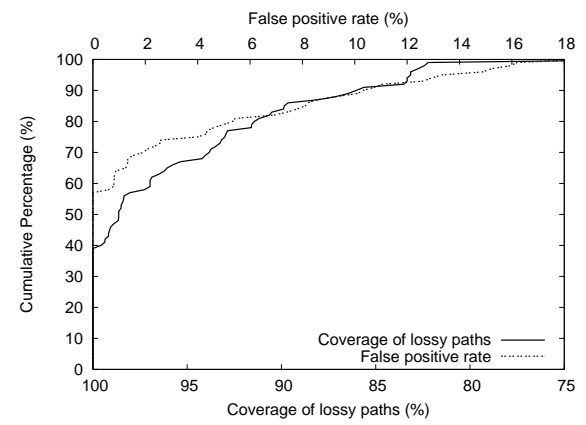

Figure 8: Cumulative percentage of the coverage and the false positive rates for lossy path inference in the 100 experiments.

As in the simulations, many of the false positives and false negatives are caused by the $5 \%$ threshold boundary effect. The average absolute error across the 100 runs is only 0.0027 for all paths, and 0.0058 for lossy paths. We pick the run with the worst accuracy in coverage $(69.2 \%)$, and plot the CDFs of absolute errors and error factors in Fig. 9. Since we only use 300 packets to measure the loss rate, the loss rate precision granularity is 0.0033 , so we use $\varepsilon=0.005$ for error factor calculation. The average error factor is only 1.1 for all paths.

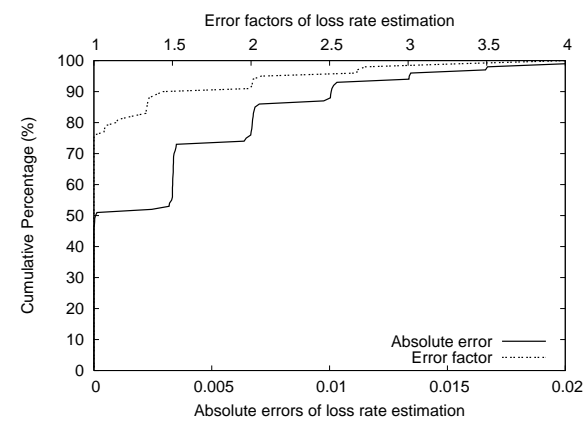

Figure 9: Cumulative percentage of the absolute errors and error factors for the experiment with the worst accuracy in coverage.

Even for the worst case, $95 \%$ of absolute errors in loss rate estimation are less than 0.014 , and $95 \%$ of error factors are less than 2.1. To further view the overall statistics, we pick 95 percentile of absolute errors and error factors in each run, and plot the CDFs on those metrics. The results are shown in Fig. 10. Notice that 90 runs have the 95 percentile of absolute errors less than 0.0133 , and 90 runs have the 95 percentile of error factors less than 2.0.

The average running time for selecting monitoring paths based on topology measurement is 0.75 second, and for loss rate calculation of all 2550 paths is 0.16 second.

\subsubsection{Topology error handling}

The limitation of traceroute, which we use to measure the topology among the end hosts, led to many topology measurement inaccuracies. As found in [34], many of the routers on the paths among PlanetLab nodes have aliases. We did not use sophisticated techniques to resolve these aliases. Thus, the topology we have is far from accurate. Furthermore, in the PlanetLab experiments, some nodes were down, 


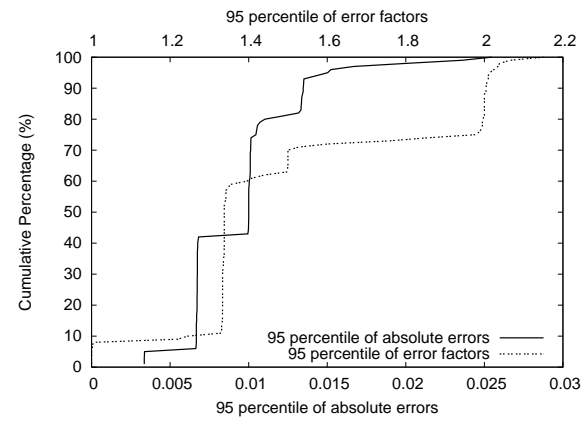

Figure 10: Cumulative percentage of the 95 percentile of absolute errors and error factors for the 100 experiments.

or were unreachable from certain nodes. Meanwhile, some routers are hidden and we only get partial routing paths. Averaging over 14 sets of traceroutes, 245 out of $51 \times 50=$ 2550 paths have no or incomplete routing information. The accurate loss rate estimation results show that our topology error handling is successful.

\section{CONCLUSIONS}

In this paper, we improve, implement and evaluate an algebraic approach [1] for adaptive scalable overlay network monitoring. For an overlay of $n$ end hosts, we selectively monitor a basis set of $O(n \log n)$ paths which can fully describe all the $O\left(n^{2}\right)$ paths. Then the measurements of the basis set are used to infer the loss rates of all other paths. Our approach works in real time, offers fast adaptation to topology changes, distributes balanced load to end hosts, and handles topology measurement errors. Both simulation and real Internet implementation yield promising results.

For even more efficient monitored path selection, we plan to investigate the use of iterative methods [32], [35] both to select rows and to compute loss rate vectors. In our preliminary experiments, the path matrix $G$ has been wellconditioned, which suggests that iterative methods may converge quickly.

\section{ACKNOWLEDGEMENT}

We thank Brian Chavez for helping with dynamic simulations. We also thank James Demmel, the anonymous reviewers and our shepherd for their valuable suggestions.

\section{REFERENCES}

[1] Y. Chen, D. Bindel, and R. H. Katz, "Tomography-based overlay network monitoring," in ACM SIGCOMM Internet Measurement Conference (IMC), 2003.

[2] Y. Chen, Towards a Scalable, Adaptive and Network-aware Content Distribution Network, Ph.D. thesis, University of California at Berkeley, Nov. 2003.

[3] D. G. Andersen et al., "Resilient overlay networks," in Proc. of ACM SOSP, 2001.

[4] T. S. E. Ng and H. Zhang, "Predicting Internet network distance with coordinates-based approaches," in Proc.of IEEE INFOCOM, 2002.

[5] S. Ratnasamy et al., "Topologically-aware overlay construction and server selection," in Proc. of IEEE INFOCOM, 2002.

[6] P. Francis et al., "IDMaps: A global Internet host distance estimation service," IEEE/ACM Trans. on Networking, Oct. 2001.

[7] Y. Chen et al., "On the stability of network distance estimation," in ACM SIGMETRICS Performance Evaluation Review (PER), Sep. 2002.
[8] Mark Coates, Alfred Hero, Robert Nowak, and Bin Yu, "Internet Tomography," IEEE Signal Processing Magazine, vol. 19, no. 3, pp. 47-65, 2002.

[9] T. Bu, N. Duffield, F. Presti, and D. Towsley, "Network tomography on general topologies," in $A C M$ SIGMETRICS, 2002.

[10] V. Padmanabhan, L. Qiu, and H. Wang, "Server-based inference of Internet link lossiness," in IEEE INFOCOM, 2003.

[11] D. Rubenstein, J. F. Kurose, and D. F. Towsley, "Detecting shared congestion of flows via end-to-end measurement," ACM Transactions on Networking, vol. 10, no. 3, 2002.

[12] Y. Shavitt, X. Sun, A. Wool, and B. Yener, "Computing the unmeasured: An algebraic approach to Internet mapping," in IEEE INFOCOM, 2001.

[13] H. C. Ozmutlu et al., "Managing end-to-end network performance via optimized monitoring strategies," Journal of Network and System Management, vol. 10, no. 1, 2002.

[14] C. Tang and P. McKinley, "On the cost-quality tradeoff in topology-aware overlay path probing," in IEEE ICNP, 2003.

[15] R. Caceres, N. Duffield, J. Horowitz, and D. Towsley, "Multicast-based inference of network-internal loss characteristics," IEEE Transactions in Information Theory, vol. 45, 1999.

[16] S. Floyd and V. Jacobson, "Random early detection gateways for congestion avoidance," IEEE/ACM Transactions on Networking, vol. 1, no. 4, 1993.

[17] N. Duffield et al., "Multicast-based loss inference with missing data," IEEE Journal of Selected Areas of Communications, vol. 20, no. 4, 2002.

[18] G.H. Golub and C.F. Van Loan, Matrix Computations, The Johns Hopkins University Press, 1989.

[19] E. Anderson et al., LAPACK Users' Guide, Society for Industrial and Applied Mathematics, Philadelphia, PA, third edition, 1999.

[20] Y. Zhang et al., "On the constancy of Internet path properties," in Proc. of SIGCOMM IMW, 2001.

[21] J.W. Demmel, Applied Numerical Linear Algebra, SIAM, 1997.

[22] H. Tangmunarunkit et al., "Network topology generators: Degree-based vs structural," in ACM SIGCOMM, 2002.

[23] M. Faloutsos, P. Faloutsos, and C. Faloutsos, "On power-law relationship of the Internet topology," in $A C M$ SIGCOMM, 1999.

[24] A. Medina, I. Matta, and J. Byers, "On the origin of power laws in Internet topologies," in ACM Computer Communication Review, Apr. 2000.

[25] R. Govindan and H. Tangmunarunkit, "Heuristics for Internet map discovery," in IEEE INFOCOM, 2000.

[26] N. Spring, R. Mahajan, and D. Wetherall, "Measuring isp topologies with rocketfuel," in ACM SIGCOMM, 2002.

[27] L. Subrmanian, S. Agarwal, J. Rexford, and R. H.Katz, "Characterizing the Internet hierarchy from multiple vantage points," in IEEE INFOCOM, 2002.

[28] G. W. Stewart, Matrix Algorithms: Basic Decompositions, Society for Industrial and Applied Mathematics, 1998.

[29] V. Paxon, "End-to-end routing behavior in the Internet," IEEE/ACM Transactions on Networking, vol. 5, no. 5, 1997.

[30] Y. Zhang, V. Paxson, and S. Shenker, "The stationarity of Internet path properties: Routing, loss, and throughput," ACIRI Technical Report, May, 2000.

[31] V. Paxon, "End-to-end Internet packet dynamics," in $A C M$ SIGCOMM, 1997.

[32] R. Barrett et al., Templates for the Solution of Linear Systems: Building Blocks for Iterative Methods, 2nd Edition, SIAM, Philadelphia, PA, 1994.

[33] PlanetLab, "http://www.planet-lab.org/,"

[34] N. Spring, D. Wetherall, and T. Anderson, "Scriptroute: A facility for distributed internet measurement," in USITS, 2003.

[35] C. Meyer and D. Pierce, "Steps toward an iterative rank-revealing method," Tech. Rep. ISSTECH-95-013, Boeing Information and Support Services, 1995. 